Rev. salud pública. 10 (4):517-528, 2008

\title{
Acoso Escolar a Estudiantes de Educación Básica y Media
}

\author{
Bullying amongst students attending state basic and middle schools \\ Edilberto Cepeda-Cuervo, Pedro N. Pacheco-Durán, Liliana García-Barco y \\ Claudia J. Piraquive-Peña
}

Departamento de Estadística, Universidad Nacional de Colombia, Bogotá. ecepedac@unal.edu.co

Recibido 25 Enero 2008/Enviado para Modificación 10 Agosto 2008/Aceptado 2 Septiembre 2008

\section{RESUMEN}

Objetivo Determinar las características y el nivel de acoso escolar en los colegios de la Localidad Ciudad Bolívar de Bogotá, Colombia.

Material y métodos La muestra se conformó con 3226 alumnos de educación básica y media, de grados sexto a once, de colegios oficiales de la localidad Ciudad Bolívar de Bogotá. Los datos obtenidos a través de la aplicación de una encuesta, en la que se estableció la frecuencia con que los alumnos son víctimas de situaciones de violencia escolar, fueron analizados estadísticamente utilizando SPSS 14.0.

Resultados Los resultados indican niveles de indiferencia, agresividad y otras formas de violencia en las escuelas. Un alto porcentaje de estudiantes es rechazado y humillado por sus compañeros. El nivel de acoso escolar resultó independiente del nivel socioeconómico de los estudiantes, pero dependiente del grado escolar en que se encuentren. Situaciones de acoso como "me dicen apodos que no me gustan" o "no cuentan conmigo para actividades de clase" son más frecuentes en los grados sexto, séptimo y octavo. Finalmente, a partir de los índices global, de intensidad y estratégico se muestra que para un alto porcentaje de estudiantes, la escuela es un espacio donde cada día trae más sufrimiento, donde se presentan múltiples situaciones de violencia que generan miedo y afectan la vida de los niños.

Conclusiones La comunidad estudiantil de la localidad de Ciudad Bolívar, en Bogotá, Colombia está afectada por un alto número de situaciones de acoso escolar.

Palabras Clave: Violencia, acoso, conducta del adolescente, Bogotá (fuente: DeCS, BIREME).

\section{ABSTRACT}

Objective Determining the characteristics and level of bullying in state schools in Ciudad Bolivar (Locality 19) in Bogotá, Colombia.

Material and methods A sample of 3226 basic education students was taken, the students being in the sixth to eleventh grades in state schools in the locality of 
Ciudad Bolivar in Bogotá. The data was collected by applying a survey in which the perception of bullying in the schools was established. SPSS 14.0 was used for statistical analysis.

Results This research took statistical results indicating the level of indifference, aggressiveness and other kinds of violence in the schools. The initial conclusion was that a high percent of students were rejected and humiliated by their peers. The level of bullying depended on the students' scholastic level rather than their socioeconomic level. Bullying situations such as giving students nicknames which they did not like or not including them in class activities were more frequent in the sixth, seventh and eighth grades. Overall, intensity and strategic indices revealed that school is a place where each day brings more suffering, where many violent situations may arise thereby making students feel afraid and that this affects the children's life for a high percentage of the students.

Conclusions The student community from the locality of Ciudad Bolivar in Bogotá, Colombia, is affected by a high number of bullying situations.

Key Words: School, violence, adolescent behaviour, Bogotá (source: MeSH, NLM).

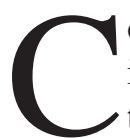

on frecuencia la violencia en la escuela se asocia a situaciones como indisciplina, robos, peleas y destrucción del material y de la infraesde las instituciones educativas. Este estudio, sin embargo, tiene como objetivo el estudio de otras formas de violencia denominadas acoso escolar, las cuales no son de fácil percepción (1).

El acoso escolar es un tipo de violencia que se manifiesta por agresiones sicológicas, físicas o sociales, repetidas, que sufre un niño en el entorno escolar por sus compañeros. Para distinguir el acoso escolar de otras acciones violentas, como por ejemplo, una pelea entre compañeros, es necesario reconocer dos características que identifican el acoso escolar. La primera es la existencia intrínseca de una relación de poder (dominio-sumisión) que tiene uno o varios agresores sobre otro que es el agredido o acosado. La segunda es que, en el acoso, las situaciones de agresión se presentan en forma reiterada. La definición de acoso escolar de Piñuel y Oñate (2) es la siguiente: “...un continuado y deliberado maltrato verbal y modal que recibe un niño por parte de otro u otros, que se comportan con él cruelmente con el objeto de someterlo, apocarlo, asustarlo, amenazarlo y que atentan contra la dignidad del niño".

Un niño acosado puede presentar depresión, temor, baja autoestima, aislamiento y sentimientos de rechazo. Estas y otras características del niño acosado afectan su vida diaria, su desarrollo personal y, en general, todas sus actividades (3). El acoso puede incluso impulsar al alumno a tomar decisiones extremas como el suicidio. 


\section{METODOLOGÍA}

Este proceso de investigación se inicia con el desarrollo de un marco teórico y de una encuesta basada en la escala de Cisneros (4), la cual tiene una fiabilidad de 0,96. Luego se desarrolla una aplicación piloto de la encuesta a una muestra de 257 alumnos de la población objetivo, en los que están incluidos estudiantes de ambos géneros y de todos los grados entre sexto y once (último grado de educación media). Los datos resultantes de esta aplicación fueron codificados y analizados con el fin de determinar claridad, coherencia y pertinencia de las preguntas, y tiempo promedio para contestar el cuestionario. Teniendo en cuenta este proceso se realizaron los cambios pertinentes para obtener la versión final de la encuesta, finalmente constituida por 22 ítems, relacionados con situaciones de acoso que se pueden presentar en del entorno escolar del estudiante. Cada uno de los ítems consta de un enunciado y tres opciones de respuesta (CF: Con Frecuencia, AV: A veces y N: Nunca), asociadas a la periodicidad de las situaciones descritas en cada uno de los enunciados de los ítems en el contexto escolar del estudiante. Entre estas posibilidades el estudiante debe seleccionar una única respuesta al contestar la encuesta (5). Finalmente, la segunda prueba piloto realizada a 97 estudiantes permitió determinar la funcionalidad de la prueba.

Muestra

La población de interés está constituida por los estudiantes de educación básica y media (grados sexto a once) de colegios oficiales de la localidad Ciudad Bolívar de Bogotá, Colombia. Está constituida por 709 cursos de colegios oficiales que atienden a más de 28000 estudiantes. Por conveniencia, los datos fueron obtenidos de una muestra de 80 cursos, 19 de grado sexto, 12 de séptimo, 12 de octavo, 10 de noveno, 10 de décimo y 17 de undécimo grado. Así, la muestra quedó constituida por 3226 estudiantes, con edades comprendidas entre 10 y 20 años, pertenecientes a los estratos socioeconómicos bajo y medio. La Tabla 1 muestra el número de estudiantes, que por grado escolar, conforman la muestra.

\section{RESULTADOS}

En esta sección se presenta la información obtenida a partir de la codificación y análisis de los datos obtenidos en la aplicación de la encuesta. La Tabla 2 muestra el porcentaje de estudiantes que "nunca”, "a veces” o "con frecuencia” son víctimas de cada una de las situaciones de acoso incluidas en la encuesta. 
Tabla 1 Discriminación de la muestra por grado escolar

\begin{tabular}{cccc}
\hline Grado escolar & Femenino & Masculino & Total \\
\hline 6 & 358 & 386 & 744 \\
7 & 288 & 201 & 489 \\
8 & 265 & 225 & 490 \\
9 & 225 & 181 & 406 \\
10 & 202 & 205 & 407 \\
11 & 414 & 276 & 690 \\
\hline Total & 1752 & 1474 & 3226 \\
\hline
\end{tabular}

Tabla 2. Porcentajes de alumnos victimas de situaciones de acoso

\begin{tabular}{|c|c|c|c|}
\hline \multirow[b]{2}{*}{ PREGUNTAS } & \multicolumn{3}{|c|}{ FRECUENCIA } \\
\hline & $\begin{array}{r}N \\
(\%)\end{array}$ & AV & CF \\
\hline Me obligan a realizar cosas que no quiero & 55,6 & 24,4 & 20 \\
\hline $\begin{array}{l}\text { Me obligan a hacer cosas que ponen en peligro mi salud } \\
\text { a propósito }\end{array}$ & 70.8 & 18.6 & 10,6 \\
\hline Le prohiben a otros hablar conmigo & 57,4 & 241 & 18,5 \\
\hline No hablan conmigo. & 48,7 & 35,5 & 15,8 \\
\hline Menosprecian mi trabajo, no importa lo que haga o diga & 62,1 & 29,1 & 8,8 \\
\hline $\begin{array}{l}\text { Hacen criticas y reproches por cualquier co sa que haga } \\
\text { o decisión que tome }\end{array}$ & 40,5 & 40.7 & 18,8 \\
\hline Me humillan y desprecian en público & 58,5 & 23,7 & 17,8 \\
\hline No cuentan conmigo para realizar actividades de la clase & 45,9 & 33,9 & 20,2 \\
\hline Cambian malintencionadamente lo que digo o hago & 49,9 & 28,4 & 21,7 \\
\hline Me culpan de todo lo malo que pasa & 56,9 & 28,9 & 14,2 \\
\hline Hacen burlas o bromas de mi & 41,4 & 40,7 & 17,9 \\
\hline Me amenazan de manera verbal o mediante gestos & 56,9 & 27,0 & 16,1 \\
\hline Me ignoran o excluyen & 60,4 & 25,9 & 13,7 \\
\hline Me interrumpen continuamente impidiendo expresarme & 47,9 & 33.7 & 18,4 \\
\hline Impiden comunicarme & 59,2 & 25,6 & 15,4 \\
\hline Hacen bromas crueles acerca de mi aspecto físico & 52,5 & 36,0 & 11,5 \\
\hline Me lanzan objetos & 55,9 & 28,9 & 15,2 \\
\hline Esconden mis objetos personales & 46,1 & 37,2 & 16,7 \\
\hline Dañan mis objetos personales & 60,3 & 31,6 & 8,1 \\
\hline Me quitan las onces & 74,5 & 13,0 & 12,5 \\
\hline Me inducen a actuar violentamente & 59,8 & 26,4 & 13,8 \\
\hline Me dicen apodos que no me gustan & 43,9 & 30,0 & 26,1 \\
\hline
\end{tabular}

Para ilustrar cómo debe interpretarse esta Tabla, damos a continuación algunos ejemplos. Con relación a los ítems "No cuentan conmigo para realizar actividades de clase” y "No hablan conmigo", que indican exclusión social, sólo un porcentaje de estudiantes menor de 50 \% no se ha sentido afectado por el fenómeno de exclusión en cada una de estas situaciones de acoso. Con relación a la primera, el 33.9\% se ha sentido excluido "algunas veces” y el 20,2 \%, “con frecuencia”. Con relación a la segunda, el 35,5 \% se ha sentido excluido "algunas veces” y el 15,8 \%, “con frecuencia”. Al analizar el ítem "Hacen bromas crueles acerca de mi aspecto físico", el 52,5 \% de los estudiantes no se ha sentido afectado por esta situación de acoso; el 36 \% “algunas veces” y el 11,5 
\%, “con frecuencia”. Con relación a "Me dicen apodos que no me gustan", el 43,9 \% se ha sentido afectado por esta situación de acoso; el 30,4 \% "algunas veces” y el 26,1 \% “con frecuencia”.

Considerando cada una de las situaciones de acoso registradas en la prueba se encuentra que, con un nivel de significancia del $1 \%$, ninguna de las situaciones de acoso escolar presentadas en la encuesta depende del estrato socioeconómico de los estudiantes. Esto significa que en todos los estratos considerados en este estudio, estrato 0 y 1 , estrato 2 y estrato 3 , las situaciones de acoso se presentan con la misma intensidad y frecuencia.

Excepto por las situaciones de acoso "No hablan conmigo", "No cuentan conmigo para realizar actividades de la clase", "Me culpan de todo lo malo que pasa” y "Me dicen apodos que no me gustan", en un nivel de significancia del 1\%, ninguna de las situaciones de acoso presentadas en la encuesta dependen del grado escolar. Esto significa que excepto en las situaciones antes mencionadas, las modalidades de acoso presentadas en la encuesta ocurren en todos los grados escolares con la misma intensidad. Las Tablas 3 y 4 muestran los porcentajes de alumnos afectados "a veces” y "con frecuencia” por estas cuatro situaciones de acoso.

Tabla 3. Descripción de situaciones "No hablan conmigo" y "No cuentan conmigo para actividades de clase" a través del grado escolar

\begin{tabular}{|c|c|c|c|c|c|c|}
\hline \multirow{2}{*}{$\begin{array}{l}\text { Grado } \\
\text { escolar }\end{array}$} & \multicolumn{3}{|c|}{ No hablan conmigo } & \multicolumn{3}{|c|}{$\begin{array}{c}\text { No cuentan conmigo para } \\
\text { actividades de clase }\end{array}$} \\
\hline & Nunce & $\begin{array}{c}\text { A } \\
\text { veces }\end{array}$ & $\begin{array}{c}\text { Con } \\
\text { frecuencia }\end{array}$ & Nunca & $\begin{array}{c}\text { A } \\
\text { veces }\end{array}$ & $\begin{array}{c}\text { Con } \\
\text { frecuencia }\end{array}$ \\
\hline 6 & 43,7 & 37,9 & 18,4 & 42,3 & 36,3 & 21,4 \\
\hline 7 & 44,2 & 37,4 & 18,4 & 44,0 & 36,0 & 20,0 \\
\hline 8 & 49,2 & 35,3 & 15,5 & 43,7 & 32,4 & 23,9 \\
\hline 9 & 47,8 & 39,2 & 13,1 & 44,8 & 35,5 & 19,7 \\
\hline 10 & 59,7 & 25,8 & 14,5 & 55,8 & 26,5 & 17,7 \\
\hline 11 & 50,9 & 35.5 & 13,6 & 47.4 & 34.3 & 18,3 \\
\hline
\end{tabular}

Tabla 4. Descripción de situaciones "Me culpan de todo lo malo que pasa" y "Me dicen apodos que no me gustan" a través del grado escolar

\begin{tabular}{lllllll}
\hline $\begin{array}{l}\text { Grado } \\
\text { escolar }\end{array}$ & \multicolumn{2}{l}{ Me culpan de todo lo malo que pasa } & \multicolumn{3}{c}{ Me dicen apodos que no me gustan } \\
\hline & Nunca & A veces & $\begin{array}{l}\text { Con } \\
\text { frecuencia }\end{array}$ & Nunca & A veces & $\begin{array}{l}\text { Con } \\
\text { frecuencia }\end{array}$ \\
\cline { 2 - 6 } 6 & 55,4 & 29,8 & 14,8 & 40,3 & 30,4 & 29,3 \\
7 & 55,4 & 32,5 & 12,1 & 40,7 & 33,3 & 26,0 \\
8 & 55,1 & 28,8 & 16,1 & 41,2 & 30,0 & 28,8 \\
9 & 52,5 & 34,2 & 13,3 & 42,6 & 30,0 & 27,3 \\
10 & 64,9 & 21,9 & 13,3 & 55,8 & 23,1 & 21,1 \\
11 & 58,7 & 26,4 & 14,9 & 45,5 & 31,4 & 23,0 \\
\hline
\end{tabular}


Para el análisis e interpretación de estos resultados según la edad de los estudiantes, en la Tabla 5 se incluyen estimaciones de la media y de la desviación estándar, entre paréntesis, de la edad de los estudiantes según el grado escolar.

Tabla 5. Media y desviación estándar de la edad de los estudiantes

\begin{tabular}{ccccccc}
\hline Grado & 6 & 7 & 8 & 9 & 10 & 11 \\
\hline $\begin{array}{l}\text { Media } \\
\text { (años) }\end{array}$ & $12,3(1,3)$ & $12,9(1,3)$ & $13,7(1,2)$ & $14,6(1,3)$ & $15,8(1,1)$ & $16,7(1,0)$ \\
\hline
\end{tabular}

En relación con el género (femenino, masculino), no se encuentran diferencias significativas en ninguna de las situaciones consideradas en la encuesta, excepto para "Me culpan de todo lo malo que pasa", situación en la cual los estudiantes de género masculino son los más acosados. Esta situación se ilustra en la Figura 1.

Figura 1. Porcentaje de alumnos afectados por la situación de acoso "Me culpan de todo lo malo que pasa"

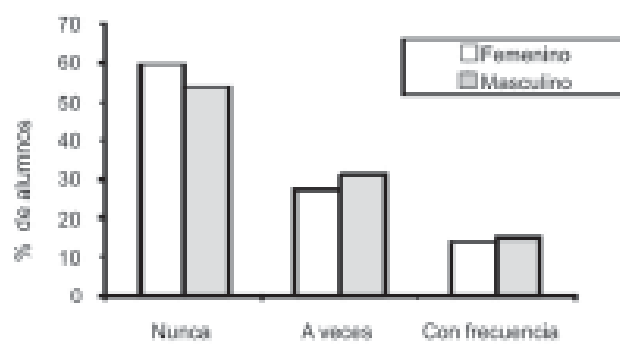

Índices de acoso escolar

Para el análisis y caracterización global del acoso escolar se define una escala numérica en la que 3 corresponde a "Con frecuencia"; 2 a "A veces"; y 1 a "Nunca”, excepto en los ítems 7, 9, 19 y 26, donde a "nunca” se asigno 3, a "a veces" 2 y a "con frecuencia” 1 . Así, en cada uno de los 22 ítems, 1 corresponde a un ambiente exento de la situación de violencia descrita, y 3 corresponde al caso donde la situación de acoso escolar, implícito en el ítem, se presenta con frecuencia. A partir de esta escala numérica se definen tres índices para el estudio del acoso, basados en la propuesta presentada de Fidalgo y Piñuel (4).

Índice global

El primer índice, denominado índice global (IG), se calcula para cada estudiante y es definido como el promedio de la puntuación de sus ítems. Este índice toma valores en una escala continua entre 1 y 3 , donde 1 indica que en el contexto escolar del estudiante no se presenta ninguna de las conductas de 
acoso registradas en la encuesta y 3 indica que en el contexto escolar del estudiante se presentan "Con frecuencia" todas las situaciones de violencia descritas.

La Figura 2 muestra la frecuencia con que aparecen en la muestra los valores posibles del IG. Haciendo una partición del rango del IG, definimos los siguientes niveles de acoso escolar: nivel I para valores del índice estratégico en el intervalo 1-1,5, nivel II para valores del IG en el intervalo 1,5-2, nivel III para valores del IG en el intervalo 2-2,5 y IV para valores del IG en el intervalo 2,5-3.

Figura 2. Distribución de frecuencia del índice global

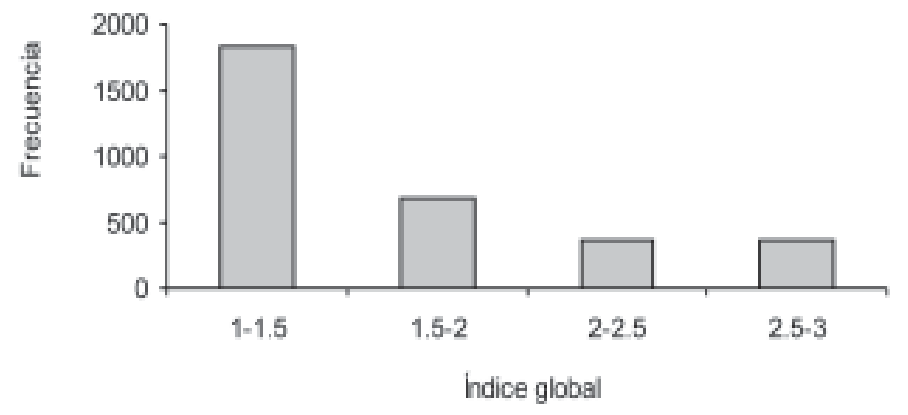

Con los niveles de acoso así definidos, encontramos 1834 (56,9 \%) estudiantes en el nivel I, 672 (20,8 \%) en un nivel II, 367 (11,4\%) en el nivel III y 353 $(10,4 \%)$ en el nivel IV. Esto indica que existe un alto número de estudiantes, mayor al 21,8 \%, para los cuales la escuela se ha convertido en un espacio donde son maltratados, donde todos los procesos de desarrollo personal y social se encuentran en riesgo. En estos casos, valdría la pena preguntarnos cuál es el nivel de calidad de la educación y si esta puede ser evaluada, como lo hacen las entidades oficiales, únicamente por el nivel de rendimiento en lenguaje, matemáticas o ciencias naturales.

Índice estratégico

El segundo índice de violencia se denomina índice estratégico (IE) y se define como el número de respuestas distintas de 1. En nuestro caso, toma valores entre 0 y 22 e indica el número de conductas de acoso escolar experimentadas por el estudiante en su escuela o colegio. Por ejemplo, si el IE de un estudiante es 12, significa que en su escuela el alumno es víctima de 12 de las 
situaciones de acoso explicitadas en la encuesta. En este estudio 248 estudiantes reportaron no sufrir ninguna de las situaciones de acoso; 149 ser víctimas de una situación, 181 de dos, como se indica en la Figura 3.

Figura 3. Distribución de frecuencia del índice estratégico

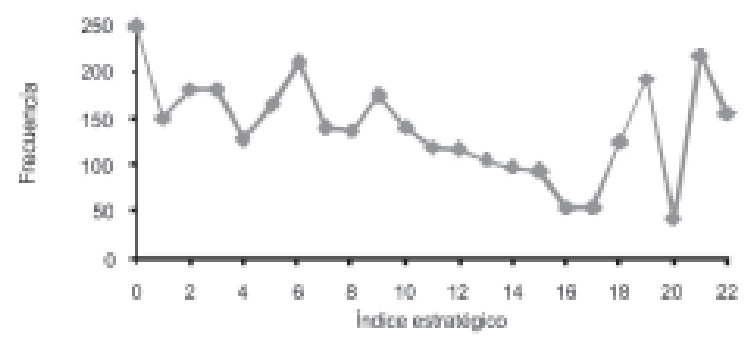

A partir de este índice, se puede observar el alto número de situaciones violentas a las que se encuentran sometidos algunos estudiantes. Por ejemplo, 156 estudiantes reportaron ser víctimas de 22 de las situaciones de violencia; 218 ser víctimas de 21; 43 estudiantes de 20 y 193 de 19.

La Tabla 6 muestra que, en general, los alumnos sufren de múltiples situaciones de acoso. El 14,5 \% de los estudiantes reportaron ser víctimas de 16 a 20 de las 22 situaciones de acoso y el 11,4 \% de 21 o 22 .

Tabla 6. Porcentaje de alumnos según el número de situaciones de acoso

\begin{tabular}{|c|c|c|c|c|c|}
\hline $\begin{array}{l}\text { Número de } \\
\text { Situaciones de scoso }\end{array}$ & 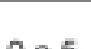 & $f \wedge-10$ & 15 & 20 & ר? \\
\hline $\begin{array}{l}\text { Porcentaje } \\
\text { de alumnos }\end{array}$ & 324 & 217 & 210 & 14.5 & 114 \\
\hline
\end{tabular}

Índice intensidad

El tercer índice se denomina índice de intensidad (II) y se define como una función del promedio de la puntuación de los ítems con respuesta diferente de "Nunca=1". Así, el II es igual a 1 si el estudiante no sufre ninguna de las situaciones de acoso escolar descritas en la encuesta. En otro caso el II toma valores en una escala continua de 2 a 3 . Un II=2.3 indica que de las situaciones de violencia que afectan al estudiante, el $30 \%$ se presentan "Con frecuencia" y $70 \%$ "A veces”. Un II=3 indica que de las situaciones de acoso que afectan a un estudiante el $100 \%$ las sufre "Con frecuencia”. 
El índice de intensidad toma valores entre 1 y 3 . Basados en el II definimos 4 niveles de acoso escolar. El primer nivel corresponde a ausencia de situaciones de acoso. En el segundo se ubican estudiantes para los cuales las situaciones de acoso se presentan "A veces" mientras que en los niveles 3 y 4 se ubican estudiantes para los cuales la frecuencia con que se presentan las situaciones de acoso es mayor. De acuerdo con lo anterior, en el nivel uno se encuentran 248 estudiantes (7,7 \%); en el nivel 2 se hallan 1313 estudiantes (40,8\%). En un nivel alto de acoso escolar se encuentran 1164 estudiantes (36\%) y en un nivel muy alto 501 estudiantes (15,5\%).

El valor del II es mayor que o igual al del IG. Una diferencia muy grande entre el II y el IG indican que el estudiante sufre pocas conductas de violencia, pero estas ocurren con una alta frecuencia.

\section{Mediana de los índices de acoso}

Debido a la asimetría de las distribuciones de los índices, se seleccionó la mediana como medida de tendencia central con el fin de observar el comportamiento de cada uno de los índices de violencia intrafamiliar por grado y por género.

Mediana del índice global

La Figura 4 muestra la mediana del IG por grado y género. Con relación al IG correspondiente al género femenino, el mayor valor de la mediana del índice global se encuentra en grado octavo. Luego, en orden decreciente, se encuentran los valores de la mediana del IG correspondientes a los grados séptimo y noveno, seguidos de la mediana del IG de grado sexto. Esto indica que en estos cursos se encuentra un mayor porcentaje de estudiantes de género femenino que sufren con mayor frecuencia las situaciones de acoso escolar descritas en la encuesta. En este caso, para los grados 10 y 11, la mediana del índice global es significativamente menor. Se observa que a partir de grado noveno la tendencia de la moda del IG para el género femenino es decreciente.

Para el género masculino la mediana del IG de acoso escolar no presenta explícitamente ninguna tendencia. La interpretación de los índices puede referirse a las edades, si tenemos en cuenta la media de las mismas en cada uno de los grados. 
Figura 4. Mediana Índice global

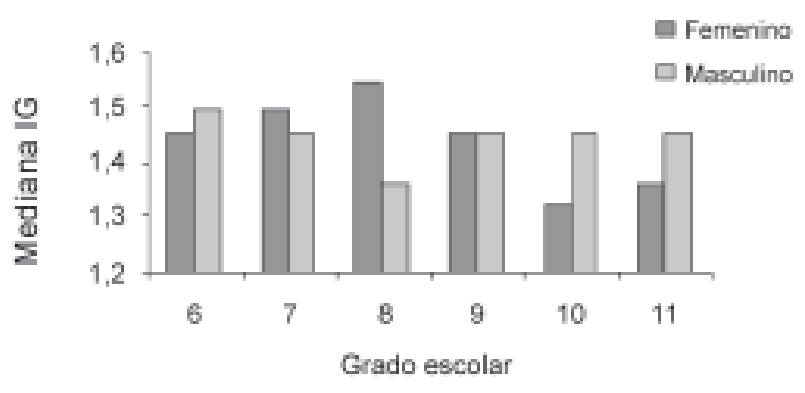

Mediana del Índice de Intensidad

La Figura 5 muestra la mediana del índice de intensidad por grado y por género. El comportamiento del índice de intensidad a través de los grados indica que los estudiantes de grado sexto, séptimo y octavo sufren con mayor intensidad de las situaciones de acoso escolar. Esto significa que, en estos grados, se presentan situaciones de acoso en un alto número de estudiantes. Sin embargo, el II muestra una tendencia decreciente a medida que aumenta el grado escolar.

Figura 5. Mediana Índice de intensidad

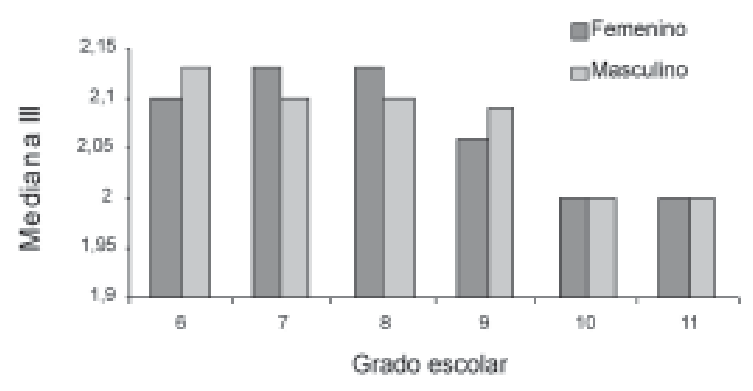

En general hay una diferencia entre el IG y el II en los diferentes grados y por géneros. Las Figuras 5 y 6 muestran que, en general, un mayor número de situaciones de acoso se presentan en los grados 6, 7, 8 y 9, especialmente cuando la comparación se hace con respecto a los grados escolares 10 y 11 .

Mediana del Índice Estratégico

En la Figura 6 se muestra la mediana del índice estratégico (IE) por género para cada uno de los grados en que se desarrolló este proceso investigativo. En general no se presentan diferencias del IE ni por grado, ni por género. Sin 
embargo, el nivel de acoso escolar es preocupante en todos los grados y géneros, pues la mediana del número de situaciones violentas que afrontan es siempre mayor que 7.

Figura 6. Mediana Índice estratégico

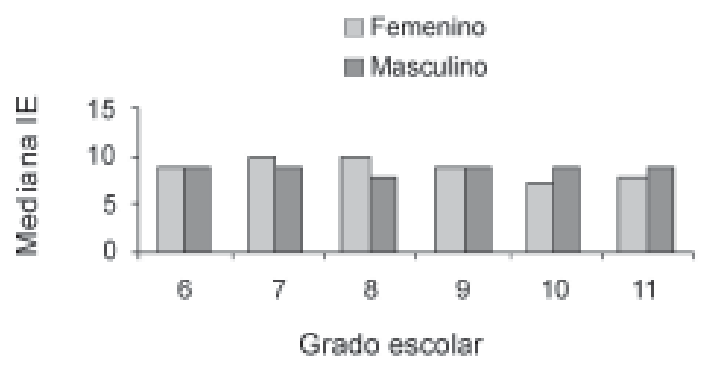

DISCUSION

En este artículo se hace un estudio de algunas situaciones de acoso escolar que afectan a estudiantes de los grados 6 a 11 de los colegios oficiales de Ciudad Bolívar, en Bogotá, Colombia. La Tabla 1 muestra que un alto porcentaje de alumnos es victima de la violencia en la escuela. Dado que investigaciones como las desarrolladas en Brasil (6) y en Estados Unidos (7), entre otras, muestran alta presencia de violencia escolar en la escuela, se concluye que este es un fenómeno global que afecta diversas culturas y diferentes sectores sociales.

El comportamiento de los índices global, específico y de intensidad muestran que la comunidad estudiantil de esta localidad está afectada por un alto número de situaciones de acoso, que se presentan con frecuencia en los colegios oficiales de este sector. Se encuentran altos índices de acoso escolar que pueden tener influencia significativa en los orígenes de las situaciones de violencia y de deserción escolar que caracterizan el sector de Ciudad Bolívar (8).

Este artículo pone en evidencia una problemática que puede estar presentándose en múltiples regiones del país y que, como se verá en otros artículos, es un factor que, además de impedir el buen rendimiento escolar de los estudiantes (9), es el origen de múltiples situaciones de ausentismo (10) y deserción escolar y, en general, de situaciones que deterioran la condición de vida de los niños y jóvenes. 
Finalmente, dado que la violencia es uno de los factores que más influye en la baja calidad de la enseñanza, de una u forma todos somos victimas de este fenómeno (11). Sin embargo, a pesar de todo, en Colombia no existe una política clara de intervención y prevención de este fenómeno, que ni siquiera es considerado en los procesos de evaluación de la calidad de la educación •

\section{REFERENCIAS}

1. Cepeda-Cuervo E, Moncada-Sáncez E, Alvarez V. Intra-family violence affecting students attending basic- and middle-schools in Bogotá. Rev. Salud Pública (Bogotá) 2007; 9(4): 516-528.

2. Piñuel I, Oñate A. Informe Cisneros VII "violencia y acoso escolar en alumnos de primaria, eso y bachiller" [Internet]. Informe preliminar. Instituto de Innovación Educativa y Desarrollo Directivo. Disponible en: www.acosoescolar.com. Consultado: Agosto 2008.

3. Cabrera E. Palabras que dejan huella: violencia en la escuela a través del discurso. Revista iberoamericana 2005; 37: 49-54.

4. Fidalgo A. y Piñuel I. La escala Cisneros como herramienta de valoración del Mobbing. Psicothema 2004; 4: 615-624.

5. Cepeda E. Factores asociados al logro cognitivo en Matemática. Revista de Educación 2005; 336: 503-514.

6. Abramovay M, Das Graças RM. Violence in Schools. Brasília: UNESCO; 2002.

7. DeVoe J, Peter K, Kaufman P, Miller A, Noonan M, Snyder T, et al. Indicators of School Crime and Safety: NCES. U.S. Department of Education, Washington, D.C.; 2004.

8. Morotti A, Roberts W. The bully as victim: Understanding bully behaviors to increase the effectiveness of interventions in the bully-Victim dyad. American Counseling Association 2000; 4: 148-156.

9. Bowen N, Bowen G. Effects of crime and violence in neighborhoods and schools on the school performance of adolescents. Journal of Adolescent Research 1999; 14: 319-342.

10. Grogger J. Local Violence and Educational Attainment. Journal of Human Resources 1997; 32(4): 659-682.

11. César del Picchia de Araujo Nogueira, R. A prática de violência entre pares: o bullyng nas escolas. Revista Iberoamericana 2005;37: 93-102. 